\title{
Multilinguales
}

\section{L'opposition accompli / inaccompli en wolof contemporain}

The Perfective and Imperfective Opposition in Contemporay Wolof

\section{Mamadou Cissé}

\section{OpenEdition}

1 Journals

\section{Édition électronique}

URL : http://journals.openedition.org/multilinguales/1301

DOI : 10.4000/multilinguales. 1301

ISSN : 2335-1853

\section{Éditeur}

Université Abderrahmane Mira - Bejaia

\section{Édition imprimée}

Date de publication : 1 juin 2015

Pagination : 147-167

ISSN : 2335-1535

\section{Référence électronique}

Mamadou Cissé, «L'opposition accompli / inaccompli en wolof contemporain », Multilinguales [En ligne], 5 | 2015, mis en ligne le 01 juin 2015, consulté le 17 septembre 2019. URL : http:// journals.openedition.org/multilinguales/1301; DOI : 10.4000/multilinguales.1301

Ce document a été généré automatiquement le 17 septembre 2019.

\section{(i) 8

Multilinguales est mise à disposition selon les termes de la Licence Creative Commons Attribution Pas d'Utilisation Commerciale - Pas de Modification 4.0 International 


\title{
L'opposition accompli / inaccompli en wolof contemporain
}

The Perfective and Imperfective Opposition in Contemporay Wolof

\author{
Mamadou Cissé
}

1 Par définition, l'aspect se distingue du temps linguistique par sa neutralité dans la deixis temporelle et correspond à la manière de considérer le procès dans son déroulement temporel. Il renvoie à la façon dont la situation est représentée en ellemême. Il est défini par Feuillet, J. (2006: 86) en ces termes: "On peut définir l'aspect comme la manière dont on envisage le déroulement d'un procès dans le temps ».

2 La polarité aspectuelle caractéristique du wolof est l'opposition de phase qui est d'ailleurs la plus répandue dans les langues. Elle comporte deux pôles: l'aspect accompli et l'aspect inaccompli. Nous avons porté notre choix sur le couple terminologique accompli / inaccompli parce que l'aspect en wolof est un aspect grammatical, c'est-à-dire un aspect dont le signifié renvoie à celui d'un tiroir verbal et est défini en tant que rapport entre le déroulement du procès et un repère temporel qui lui est extérieur. Cette catégorie aspectuelle s'oppose à l'aspect dans les langues slaves qui est un aspect lexical, c'est-à-dire un aspect opposant un procès comportant un seuil à franchir et un procès dépourvu de toute limitation de cet ordre. Selon Creissels :
Le système verbal slave peut se comprendre comme un cas extrême de systématisation d'une
distinction de verbes dont le signifié inclut la notion de franchissement d'un seuil (les verbes
dits "perfectifs") et d'autres qui n'ont pas cette implication (les verbes dits "imperfectifs ").
(1995: 175)

L'accompli exprime une action parvenue à son terme ou un état achevé. Il se situe soit dans l'actuel soit dans l'inactuel. En dehors de l'action déjà réalisée ou de l'état achevé, l'aspect accompli peut être utilisé pour la description d'un état ou la caractérisation d'un être ou d'une chose. En wolof, il est exempt de toute marque formelle. En français, l'aspect accompli est exprimé par les temps composés pour les verbes d'action et par le présent pour les verbes d'état.

4 L'inaccompli correspond à l'aspect de l'action toujours en cours de réalisation, celle qui n'est pas encore parvenue à son terme. En wolof, il apparaît formellement comme la 
catégorie marquée de l'opposition aspectuelle. Il est caractérisé, d'un point de vue morphologique, par une forme généralement isolée ou par sa variante suffixale et s'oppose, de ce fait, à l'accompli, caractérisé par une absence de marque morphologique. L'inaccompli est traduit en français par le présent pour l'action en cours ou le fait permanent et par le futur pour l'action projetée.

5 Notre démarche s'articule sur quatre axes:(1) la présentation morphologique du morphème de l'inaccompli, (2) l'illustration et l'analyse du contraste accompli / inaccompli à travers les paradigmes de conjugaison, (3) la combinaison de l'inaccompli avec d'autres modalités, (4) les valeurs de l'inaccompli.

\section{Morphologie}

6 En wolof contemporain, les marques aspectuelles caractéristiques de l'inaccompli, apparaissent sous différentes formes selon le modèle de conjugaison ou le nombre. Elles correspondent à la forme di et à ses variantes $d-,-y$, et $-a$, attestée plus couramment dans les parlers de la région du Fleuve (St- Louis et Mauritanie), le parler Lébou et également les parlers du Sud (Saloum et Gambie). La forme di, autonome en règle générale, est, soit antéposée au thème verbal (2.1.1.1.), soit préfixée à une marque personnelle (2.1.1.2.). $-Y$ et $-a$ sont respectivement suffixés aux marques personnelles et à la modalité présentative alors que le marqueur $d$ - est préfixé à d'autres modalités grammaticales telles que les morphèmes du passé. Si le complément d'objet ou de lieu subit une indexation, le marqueur de l'inaccompli peut se déplacer sur le pronom (13b. et 15b.).

Les paradigmes ou modèles de conjugaison se définissent comme des ensembles de formes verbales qui sont structuralement composées de verbes, de marques de personne, de nombre, de mode, de temps et d'aspect. Dans le cadre de ces illustrations, nous nous proposons de présenter les modèles de conjugaison de deux catégories aspectuelles (accompli et inaccompli) et des formes affirmative et négative.

\section{Accompli et inaccompli affirmatifs}

7 Les modèles de conjugaison qui feront l'objet d'une analyse de l'accompli et à l'inaccompli sont ceux du mode indicatif (2.1.1), du mode injonctif (2.1.2.) et du mode subordinatif (2.1.3.).

\subsection{Le mode indicatif}

Le mode indicatif renvoie au mode global de l'énoncé déclaratif simple. Dans ce mode, l'accompli traduit une action achevée ou un état acquis tandis que l'inaccompli correspond à une action inachevée. Il comporte six modèles : le narratif (2.1.1.1.), le parfait (2.1.1.2.), l'emphatique du sujet (2.1.1.3.), l'emphatique du verbe (2.1.1.4.), l'emphatique du complément (2.1.1.5.) et le présentatif (2.1.1.6.).

\subsubsection{Le narratif}

9 Le narratif est un modèle de conjugaison non emphatique employé en récit. Le matériel morphologique caractéristique de ce paradigme réside dans le segment nasal initial $\mathrm{m}$ pour les 1 sg et 3sg, $n$ - pour les $1 \mathrm{pl}$ et $3 \mathrm{pl}$ et la prénasale $n g$ - pour les 2 sg et $2 \mathrm{pl}$. 
Par rapport aux autres paradigmes du système verbal wolof, le narratif se particularise par :

- sa simplicité morphologique qui justifie d'ailleurs son appellation de «minimal» par E. Church (1981) et sa qualification de paradigme « inanalysable » par S. Robert (1989),

- son statut de véritable pronom à la troisième personne du singulier et du pluriel. Dans ces personnes, la marque pronominale est susceptible de remplacer un syntagme ou un lexème sujet.

Les formes personnelles du narratif se présentent ainsi sous la forme de marques pronominales antéposées au lexème verbal.

\begin{tabular}{|c|c|}
\hline Singulier & Pluriel \\
\hline $\begin{array}{l}\text { 1. ma } \\
\text { 2. } \mathrm{nga} \\
\text { 3. } \mathrm{mu}\end{array}$ & ñu ngeen ñu \\
\hline Accompli & Inaccompli \\
\hline $\begin{array}{l}\text { 1. ma bay gerte } \\
\text { "Je cultive l'arachide» } \\
\text { 2. ngeen bay gerte } \\
\text { «Vous cultivez } \\
\text { l'arachide» }\end{array}$ & $\begin{array}{l}\text { may bay gerte } \\
\text { «Je suis en train de cultiver l'arachide » ngeen di } \\
\text { bay gerte } \\
\text { «Vous êtes en train de cultiver l'arachide» }\end{array}$ \\
\hline
\end{tabular}

10 On observe ici que, pour le narratif, l'aspect accompli est une catégorie grammaticale exempte de toute marque formelle.

Quant à la forme inaccomplie, elle est marquée, au niveau du narratif, par le morphème di. Celle-ci est postposée aux marques personnelles qui sont antéposées au lexème verbal. L'opposition marques personnelles «accompli»/ marques personnelles « inaccompli » peuvent être illustrées de la façon suivante :

\begin{tabular}{|l|l|}
\hline \multicolumn{1}{|c|}{ Accompli } & Inaccompli \\
\hline $\begin{array}{l}\text { Ngeen xaar Omar } \\
\text { « Vous attendez Omar » }\end{array}$ & $\begin{array}{l}\text { Ngeen di xaar Omar } \\
\text { « Vous êtes en train d'attendre Omar » }\end{array}$ \\
\hline
\end{tabular}

\subsubsection{Le parfait}

11 Le parfait est formellement marqué par l'élément na antéposé ou amalgamé aux marques personnelles. Il renvoie à un procès dont l'achèvement est supposé connu. Contrairement à l'armature syntagmatique dominante en wolof où les marques personnelles précèdent le lexème verbal, les indices personnels du parfait se positionnent après le radical verbal, comme dans l'exemple ci-après :

12

Gis nañu Omar - « Nous avons vu Omar » 


\begin{tabular}{|l|l|}
\hline \multicolumn{1}{|c|}{ Accompli } & \multicolumn{1}{c|}{ Inaccompli } \\
\hline $\begin{array}{l}\text { Sant nga Yàlla } \\
\text { « Tu as remercié Dieu » Dinga sant } \\
\text { Yàlla } \\
\text { «Tu remercieras Dieu » }\end{array}$ & $\begin{array}{l}\text { dinga sant Yàlla ( danga sant Yàlla) } \\
\text { « Tu as remercié Dieu » Dinga di sant } \\
\text { Yàlla } \\
\text { "Tu as l'habitude de remercier Dieu » }\end{array}$ \\
\hline
\end{tabular}

13 Le marquage zéro caractérise le parfait de l'accompli. Ce dernier traduit un procès achevé, ce qui n'est pas le cas de l'inaccompli qui porte une marque morphologique. Cette marque apparaît sous deux formes de valeurs grammaticales différentes :

- la forme isolée di, qui se charge d'une valeur prospective et qui peut être préfixée par rapport à toutes les marques de personne du parfait,

- elle peut apparaître deux fois dans la forme verbale : préfixée par rapport à la marque de personne, postposée à cette dernière et antéposée au lexème verbal. Dans cette construction, la marque de l'inaccompli a une valeur habituelle.

\subsubsection{L'emphatique du sujet}

14 L'emphatique du sujet est une modalité qui sert à la mise en relief du sujet et à son identification parmi tous les sujets possibles dans un énoncé. Sa traduction en français correspond à l'expression « c'est X qui... ». Le marqueur modal de l'emphatique du sujet est le segment - $a$ postposé au sujet de l'énoncé qui est soit une unité lexématique, soit une marque personnelle caractéristique de cette modalité. Les indices personnels de l'emphatique du sujet se présentent comme suit :

\begin{tabular}{|l|l|}
\hline \multicolumn{1}{|c|}{ Singulier } & \multicolumn{1}{c|}{ Pluriel } \\
\hline $\begin{array}{l}\text { 1. maa } \\
\text { 2. yaa } \\
\text { 3. moo }\end{array}$ & ñoo yéena ñoo \\
\hline \multicolumn{1}{|c|}{ Accompli } & \multicolumn{1}{c|}{ Inaccompli } \\
\hline $\begin{array}{l}\text { yaa maye nag wi } \\
\text { «C'est toi qui as offert la vache » }\end{array}$ & $\begin{array}{l}\text { yaay maye nag wi } \\
\text { «C'est toi qui vas offrir la vache » }\end{array}$ \\
\hline
\end{tabular}

15 Comme pour le narratif, pour l'emphatique du sujet le morphème de l'inaccompli est facilement identifiable dans la forme verbale en raison de sa position détachée de la marque de personne qu'il suit immédiatement et du radical verbal qu'il précède immédiatement. Quant à l'accompli, il apparaît sans aucune marque formelle.

\subsubsection{L'emphatique du verbe}

16 Le morphème caractéristique de l'emphatique du verbe est le marqueur da. C'est un schème énonciatif où le focus porte sur le verbe, qu'il s'agisse d'un verbe d'action ou d'état. Il explique ou élargit un énoncé précédent d'où sa qualification de «modalité 
explicative» par certains spécialistes. Ses indices de personne sont antéposés au lexème verbal et se présentent comme suit:

\begin{tabular}{|c|c|}
\hline Singulier & Pluriel \\
\hline $\begin{array}{l}\text { 1. dama } \\
\text { 2. danga } \\
\text { 3. dafa }\end{array}$ & danu dangeen dañu \\
\hline Accompli & Inaccompli \\
\hline $\begin{array}{l}\text { dama naan ndox } \\
\text { «(C'est que) je bois de l'eau » Dangeen } \\
\text { naan sëng }\end{array}$ & $\begin{array}{l}\text { damay naan ndox } \\
\text { «(C'est que) Je suis en train de boire } \\
\text { de l'eau » }\end{array}$ \\
\hline
\end{tabular}

17 Le morphème de l'inaccompli pour l'emphatique du verbe correspond à l'élément di et à sa variante $-y$. Le marqueur aspectuel di est placé entre la marque de personne ngeen (deuxième personne du pluriel) et le radical verbal. La variante $-y$ est suffixée aux autres personnes. Elle est physiquement apparente si sa voyelle initiale ne fait pas l'objet d'une contraction avec la voyelle finale du morphème personnel. La forme danuy / dañuy des première et troisième personnes du pluriel résulte de la fusion des formes pleines de ces deux personnes, danu / dañu, et l'allomorphe -y de l'inaccompli.

\subsubsection{L'emphatique du complément}

L'emphatique du complément complète le triptyque des constructions emphatisantes qui constituent, de ce fait, un système en wolof. Il porte l'emphase sur le complément ${ }^{1}$ pour l'expression d'une identification.

19 Il est caractérisé, sur le plan formel, par la marque distinctive -la qui représente le monème du complément focalisé et permet la construction d'énoncés à prédicat nonverbal et d'énoncés à prédicat verbal. Ce morphème est repérable aux première et troisième personnes du singulier et du pluriel et est non apparent dans les deuxièmes personnes du singulier et du pluriel. Les indices de personne pour l'emphatique du complément se présentent comme suit :

\begin{tabular}{|l|l|}
\hline \multicolumn{1}{|c|}{ Singulier } & \multicolumn{1}{c|}{ Pluriel } \\
\hline $\begin{array}{l}\text { 1. laa } \\
\text { 2. nga } \\
\text { 3. la }\end{array}$ & lanu/lañu ngeen lañu \\
\hline \multicolumn{1}{|c|}{ Accompli } & \multicolumn{1}{c|}{ Inaccompli } \\
\hline $\begin{array}{l}\text { ceeb la bay } \\
\text { «C'est du riz que j'ai mangé » }\end{array}$ & $\begin{array}{l}\text { ceeb laay bay } \\
\text { «C'est du riz que je suis en train de manger » }\end{array}$ \\
\hline
\end{tabular}


Comme pour l'emphatique du verbe, l'inaccompli de l'emphatique du complément est marqué par le morphème -di qui apparaît à la deuxième personne du pluriel et sa variante $-y$, suffixée aux autres personnes.

\subsubsection{Le présentatif}

21 Le présentatif est une modalité énonciative qui saisit le procès dans son déroulement, l'action se déroule sans atteindre son terme.

Dans sa structure morphologique, le présentatif renvoie à un dispositif complexe constitué :

- des formes pronominales du narratif,

- d'un morphème $a$ préposé à $n g$ - qui marque la mise en relief du sujet,

- ng qui marque le présentatif

- et d'une marque déictique $i$ - (proximité) ou a- (éloignement) :

\begin{tabular}{|l|l|}
\hline $\begin{array}{l}\text { 1. maa ngi } \\
\text { 2. yaa ngi } \\
\text { 3. mu ngi }\end{array}$ & ñu ngi yéena ngi ñu ngi \\
\hline Accompli & \multicolumn{1}{|c|}{ Inaccompli } \\
\hline $\begin{array}{l}\text { maa ngi génn } \\
\text { « Je sors » }\end{array}$ & $\begin{array}{l}\text { maa ngiy génn } \\
\text { « Je suis en train de sortir » }\end{array}$ \\
\hline
\end{tabular}

L'opposition aspectuelle dans le présentatif est inopérante en wolof contemporain. C'est l'identité sémantique entre la nature cursive du procès présentatif et l'inachèvement dans le procès inaccompli qui neutralisent cette opposition aspectuelle. La nature cursive du procès présentatif explique l'absence de marque formelle qui distinguerait l'accompli de l'inaccompli; les deux formes se présentant de la même façon aux deux pôles aspectuels.

\subsubsection{Le mode injonctif}

23 L'injonctif renvoie au mode des ordres et des directives qui peuvent être stricts ou atténués. À chacun des deux pôles, l'aspect comporte un sens qui lui est spécifique. L'accompli traduit une action envisagée dans sa globalité, alors que l'inaccompli exprime une action envisagée dans son déroulement. L'injonctif comporte deux modèles : q l'impératif (2.1.2.1.) pour un ordre strict, et l'obligatif (2.1.2.2.) pour un ordre atténué.

\subsubsection{L'impératif}

L'impératif, ou modalité de l'ordre direct, n'implique que deux personnes :

- la deuxième personne du singulier de marque -al,

- la deuxième personne du pluriel -leen.

\begin{tabular}{|l|l|}
\hline Accompli & Inaccompli \\
\hline
\end{tabular}




\begin{tabular}{|l|l|}
\hline $\begin{array}{l}\text { Waxal dëgg gi } \\
\text { «Dis la vérité » Waxleen } \\
\text { dëgg } \\
\text { «Dites la vérité » }\end{array}$ & $\begin{array}{l}\text { dil wax dëgg gi deel }(\mathrm{di}+\mathrm{al}) \\
\text { "Aie l'habitude de dire la vérité » dileen wax dëgg } \\
\text { deeleeen } \\
\text { «Ayez l'habitude de dire la vérité » }\end{array}$ \\
\hline
\end{tabular}

25 L'inaccompli de l'impératif s'exprime par la forme di, seulement préfixée à la marque personnelle ou préfixée et reprise isolément à la deuxième personne du pluriel. L'accompli n'a pas d'expression aspectuelle formelle.

\subsubsection{L'obligatif}

L'obligatif, ou modalité de l'ordre indirect, exprime des directives, des contenus sémantiques de souhait, de recommandation, etc. Il partage le même marqueur formel que le parfait na dont il se démarque par la position antéposée de la marque personnelle, postposée au parfait. Le marqueur na est combiné avec la marque personnelle aux deuxièmes personne du singulier et du pluriel à l'obligatif alors qu'il s'élide à ces mêmes personnes au parfait. Ses marques de personne apparaissent comme suit :

\begin{tabular}{|l|l|}
\hline \multicolumn{1}{|c|}{ Singulier } & \multicolumn{1}{c|}{ Pluriel } \\
\hline $\begin{array}{l}\text { 1. naa } \\
\text { 2. nanga } \\
\text { 3. na }\end{array}$ & nanu/nañu nangeen nañu \\
\hline \multicolumn{1}{|c|}{ Accompli } & \multicolumn{1}{c|}{ Inaccompli } \\
\hline $\begin{array}{l}\text { nañu tukki njël } \\
\text { « Voyageons à l'aube » }\end{array}$ & $\begin{array}{l}\text { nanuy tukku njël } \\
\text { « Ayons l'habitude de voyager à l'aube » }\end{array}$ \\
\hline
\end{tabular}

Le marquage formel de l'inaccompli se fait à l'obligatif par le marqueur di et sa variante $-y$. La forme di est la seule marque à la deuxième personne du pluriel mais elle est interchangeable avec sa variante $-y$ pour les autres personnes. La catégorie aspectuelle « accompli » est morphologiquement non marquée.

\subsubsection{Le mode subordinatif}

Le mode subordinatif, ou "relatif», est, selon A. Dialo (1983: 60), le mode de la dépendance. Il comporte deux modèles de conjugaison, le temporel (2.1.3.1.) et l'hypothétique (2.1.3.2.), formellement marqués par les morphèmes discontinus bi... ee, ba...ee (temporel), bu...ee et su...ee (hypothétique). Ces modèles impliquent des propositions dépendantes. 


\subsubsection{Le temporel}

Le temporel a les mêmes marques personnelles que le narratif (2.1.1.1.). D'un point de vue syntaxique, les morphèmes $b i / b a$ sont antéposés à la marque de personne alors que l'élément -ee est suffixé au radical verbal.

\begin{tabular}{|l|l|}
\hline \multicolumn{1}{|c|}{ Accompli } & \multicolumn{1}{c|}{ Inaccompli } \\
\hline $\begin{array}{l}\text { bi / ba ma bindee bataaxal la } \\
\text { dem } \\
\text { «C'est lorsque j'ai écrit la lettre } \\
\text { qu'il est parti » }\end{array}$ & $\begin{array}{l}\text { bi / ba may bind bataaxal la dem } \\
\text { «C'est lorsque j'étais en train d'écrire la } \\
\text { lettre qu'il est parti » }\end{array}$ \\
\hline
\end{tabular}

$\mathrm{Au}$ temporel, les catégories aspectuelles «accompli » et «inaccompli » sont formellement marquées par des suffixes, sauf à la deuxième personne du pluriel de la forme du temporel « inaccompli » dont la marque est le morphème isolé di. En effet, le suffixe -ee, porté par le radical verbal, s'est spécialisé dans l'expression de l'antériorité, alors que la variante -y du morphème de l'inaccompli est suffixée à toutes les marques de personne à l'inaccompli. Elle est matériellement apparente si sa voyelle initiale n'a pas fait l'objet d'une contraction avec la voyelle finale du morphème personnel :

\begin{tabular}{|l|l|}
\hline \multicolumn{1}{|c|}{ Accompli } & \multicolumn{1}{c|}{ Inaccompli } \\
\hline $\begin{array}{l}\text { bi / ba ma waxee la noppi } \\
\text { «C'est lorsque j'ai parlé qu'il } \\
\text { s'est tu » }\end{array}$ & $\begin{array}{l}\text { bi / ba ma waxee la noppi } \\
\text { «C'est lorsque j'étais en train de parler qu'il } \\
\text { s'est tu » }\end{array}$ \\
\hline & \\
\hline
\end{tabular}

\subsubsection{L'hypothétique}

31 Les marques personnelles de l'hypothétique sont formellement identiques à celles du narratif (2.1.1.1.). D'un point de vue syntaxique, les morphèmes $b u / s u$ sont antéposés à la marque de personne alors que l'élément-ee est suffixé au radical verbal.

\begin{tabular}{|c|l|}
\hline Accompli & \multicolumn{1}{|c|}{ Inaccompli } \\
\hline $\begin{array}{l}\text { buñu / suñu bindee bataaxal } \\
\text { «Si nous écrivons une lettre » }\end{array}$ & $\begin{array}{l}\text { bu / su ñuy bind bataaxal } \\
\text { « Si nous sommes en train d'écrire une lettre » }\end{array}$ \\
\hline
\end{tabular}

A l'hypothétique, les catégories d'aspects accompli et inaccompli sont formellement marquées par des suffixes et la forme détachée di. En effet, le suffixe -ee, porté par le radical verbal, traduit l'hypothétique alors que l'inaccompli est marqué par la forme $d i$, notée dans les deuxièmes personnes du singulier et du pluriel, dans la troisième personne du singulier et sa variante $-y$ apparaissant pour les autres personnes. Elle est apparente à la première personne du singulier et fait l'objet d'une coalescence avec la 
voyelle finale du morphème personnel, à la première et à la troisième personne du pluriel, en raison de sa voyelle initiale.

\begin{tabular}{|c|c|}
\hline Accompli & Inaccompli \\
\hline boo / soo ...ee & boo / soo dee (di+ee) \\
\hline
\end{tabular}

\subsection{Accompli et inaccompli négatifs}

\subsubsection{Le mode indicatif}

\subsubsection{Narratif négatif}

Le narratif affirmatif n'a pas de forme négative correspondante. Il peut partager le même négatif notionnel avec le parfait (2.2.1.2.).

\subsubsection{Parfait négatif}

La négation du parfait aux deux aspects, accompli et inaccompli, correspond au négatif simple pour l'accompli et au négatif emphatique construit avec la forme isolée di pour l'inaccompli.

L'énoncé négatif simple renvoie à la négation de l'actualité du procès au moment de l'énonciation. Les marques personnelles constitutives de son paradigme sont suffixées au radical verbal et se présentent comme suit :

\begin{tabular}{|l|l|}
\hline Singulier & \multicolumn{1}{|c|}{ Pluriel } \\
\hline 1. -uma & -unu/uñu \\
2. -oo & -uleen \\
3. -ul & -uñu \\
\hline
\end{tabular}

Le négatif emphatique est inclus dans les conjugaisons emphatisantes puisqu'il permet de nier le procès en tant que notion (sans référence au temps d'énonciation) pouvant également avoir une valeur de négation de l'habitude. Il est formellement construit à partir de la consonne $d$ - de l'inaccompli di qui lui attribue, de ce fait, une valeur de futur à laquelle sont suffixées les marques personnelles du négatif simple, excepté la troisième personne du singulier qui ne porte pas la marque de personne.

\begin{tabular}{|l|l|l|}
\hline \multicolumn{2}{|c|}{ Singulier } & \multicolumn{1}{l|}{ Pluriel } \\
\hline $\begin{array}{l}\text { 1. duma } \\
\text { 2. doo } \\
\text { 3.du }\end{array}$ & \multicolumn{2}{l|}{ dunu/duñu dungeen duñu } \\
\hline Accompli = & $\begin{array}{l}\text { Négatif simple } \\
\text { Inaccompli }=\end{array}$ & $\begin{array}{l}\text { Négatif emphatique } \\
\text { /habituel }\end{array}$ \\
\hline
\end{tabular}




\begin{tabular}{|c|c|c|}
\hline $\begin{array}{l}\text { binduma } \\
\text { «Je n'ai pas écrit » } \\
\text { bindul } \\
\text { «Il n'a pas écrit » }\end{array}$ & $\begin{array}{l}\text { duma bind b/ } \\
\text { «je n'écris pas » } \\
\text { du bind/ } \\
\text { « Il n'écrit pas» }\end{array}$ & $\begin{array}{l}\text { dumay bind } \\
\text { « je n'ai pas l'habitude d'écrire » } \\
\text { duy bind } \\
\text { « Il n'a pas l'habitude d'écrire » }\end{array}$ \\
\hline
\end{tabular}

36 Au parfait, la catégorie « accompli » est portée par les marques personnelles du négatif simple qui traduisent un procès achevé. La catégorie «inaccompli », quant à elle, est doublement marquée: par la variante $d$ - de l'inaccompli, d'une part, et par le morphème di, noté à toutes les personnes et placé entre la marque de personne et le radical verbal, d'autre part. Les marques personnelles négatives apparaissent aux aspects accompli et inaccompli, sous les formes suivantes :

\begin{tabular}{|l|l|}
\hline \multicolumn{1}{|c|}{ Accompli } & \multicolumn{1}{c|}{ Inaccompli } \\
\hline $\begin{array}{l}\text { - duma dem Dakar } \\
\text { « Je ne vais pas à Dakar » }\end{array}$ & $\begin{array}{l}\text { dumay dem Dakar } \\
\text { «Je n'ai pas l'habitude d'aller à Dakar » }\end{array}$ \\
\hline
\end{tabular}

\subsubsection{L'emphatique du sujet négatif}

\begin{tabular}{|l|l|}
\hline \multicolumn{1}{|c|}{ Accompli } & \multicolumn{1}{c|}{ Inaccompli } \\
\hline $\begin{array}{l}\text { yaa bindul bataaxal } \\
\text { «C'est toi qui n'as pas écrit de } \\
\text { lettre » }\end{array}$ & $\begin{array}{l}\text { yaa dul bind bataaxal } \\
\text { «C'est toi qui n'as pas l'habitude d'écrire de } \\
\text { lettre » }\end{array}$ \\
\hline
\end{tabular}

37 A l'emphatique du sujet, l'accompli négatif est caractérisé par la forme -ul (morphème négatif + marque de personne) du négatif simple qui traduit un procès achevé. L'inaccompli négatif est formellement marqué par le bloc dul, noté à toutes les personnes et placé entre la marque de personne et le radical verbal. La forme dul est composée de la variante de l'inaccompli $d$ - et du morphème négatif -ul.

\subsubsection{L'emphatique du verbe négatif}

\begin{tabular}{|c|l|}
\hline Accompli & \multicolumn{1}{|c|}{ Inaccompli } \\
\hline $\begin{array}{l}\text { dama bindul bataaxal } \\
\text { «Je n'ai pas écrit de lettre » }\end{array}$ & $\begin{array}{l}\text { dama dul bind bataaxal } \\
\text { « Je n'ai pas l'habitude d'écrire de lettre » }\end{array}$ \\
\hline \multicolumn{2}{|c|}{ dul/duloon (passé) / daanul (habituel) } \\
\hline
\end{tabular}

A l'emphatique du verbe, l'expression de l'accompli négatif est similaire à celle de l'emphatique du sujet. L'inaccompli négatif est construit avec la forme daanul, notée à toutes les personnes et placée entre la marque de personne et le radical verbal. 
L'élément est formellement composé de la variante de l'inaccompli $d-$, du morphème aan du passé habituel et de la marque négative -ul:

\begin{tabular}{|c|c|}
\hline Accompli & Inaccompli \\
\hline dangeen ...ul & dangeen daanul \\
\hline
\end{tabular}

\subsubsection{L'emphatique du complément négatif}

\begin{tabular}{|l|l|}
\hline \multicolumn{1}{|c|}{ Accompli } & \multicolumn{1}{c|}{ Inaccompli } \\
\hline bataaxal lañu bindul & bataaxal lañu dul bind \\
"C'est (tout sauf) une lettre que & «C'est (tout sauf) une lettre que je n'ai pas \\
je n'ai pas écrite » & l'habitude d'écrire » \\
\hline
\end{tabular}

A l'emphatique du complément, l'expression de l'accompli négatif est identique à celle de l'emphatique du sujet et du verbe. La forme -ul du négatif simple, exprimant un procès déjà réalisé, est suffixée au radical verbal. L'inaccompli négatif est traduit par le morphème dul, noté à toutes les personnes et placé entre la marque de personne et le radical verbal comme à l'emphatique du sujet :

\begin{tabular}{|l|l|}
\hline Accompli & Inaccompli \\
\hline laa ...ul & laa dul \\
\hline \multicolumn{2}{|l|}{ passé laa doonul/daawul } \\
\hline
\end{tabular}

\subsubsection{Le présentatif négatif}

La modalité présentative n'a pas de forme négative en raison de son sémantisme. Les notions de présentatif et de négatif sont sémantiquement incompatibles. Cependant, ce qu'il est possible d'avoir, dans cette forme, pour exprimer la négation est, selon A. Dialo (2012 : 13), une suppléance idiomatique restreinte avec nekk « être », par exemple :

\begin{tabular}{|c|c|c|c|}
\hline $\begin{array}{l}\text { *01 - yoon-am } \\
\text { Chemin pos 3sg } \\
\text { «C'est son affaire, il a le droit de... » }\end{array}$ & $\begin{array}{l}\text { a ng-i } \\
\text { emph+prés+pro }\end{array}$ & $\begin{array}{l}\text { ci } \\
\text { part }\end{array}$ & forme positive \\
\hline
\end{tabular}

\begin{tabular}{|c|c|c|c|}
\hline $\begin{array}{l}*_{02}-\text { yoon-am } \\
\text { Chemin-pos } 3 \sin \end{array}$ & $\begin{array}{l}n e k k-u \\
\text { être-nég }\end{array}$ & $\begin{array}{l}\mathrm{Ci} \\
\text { part }\end{array} \mid$ & forme négative \\
\hline
\end{tabular}


L'énoncé yoon-am nekku ci correspond donc à l'accompli négatif du présentatif dont la forme de l'« inaccompli » est:

\begin{tabular}{|l|l|l|l|}
\hline $\begin{array}{l}\text { *03 - yoon-am } \\
\text { Chemin-pos.3sg }\end{array}$ & $\begin{array}{l}\mathrm{d}-\mathrm{u} \\
\text { inacc-neg }\end{array}$ & $\begin{array}{l}\text { ci } \\
\text { part }\end{array}$ & $\begin{array}{l}\text { nekk } \\
\text { être }\end{array}$ \\
\hline
\end{tabular}

Cette forme de l'inaccompli négatif du présentatif est traduite par la variante $d$ - de l'inaccompli qui porte la marque négative $-u$.

\subsubsection{Le mode injonctif}

41 Ce mode n'a qu'une seule forme pour l'impératif et l'obligatif :

\begin{tabular}{|l|l|l|l|l|}
\hline \multicolumn{2}{|c|}{ Accompli } & \multicolumn{2}{c|}{ Inaccompli } \\
\hline $\begin{array}{l}\text { 1. } \\
\text { 2. }\end{array}$ & $\begin{array}{l}\text { buma } \\
\text { bul } \\
\text { 3. }\end{array}$ & $\begin{array}{l}\text { bu mu } \mathrm{mu} \text { ñu buleen } \\
\text { buñu }\end{array}$ & bumay bul di bu & $\begin{array}{l}\text { bu ñuy bu leen di bu } \\
\text { muy }\end{array}$ \\
\hline
\end{tabular}

\subsubsection{L'impératif négatif}

\begin{tabular}{|l|l|}
\hline \multicolumn{1}{|c|}{ Accompli } & \multicolumn{1}{c|}{ Inaccompli } \\
\hline $\begin{array}{l}\text { bul bind bataaxal } \\
\text { « N'écris pas de lettre » }\end{array}$ & $\begin{array}{l}\text { bul di bind bataaxal } \\
\text { "N'aie pas l'habitude d'écrire de lettre » }\end{array}$ \\
\hline
\end{tabular}

\subsubsection{L'obligatif négatif ou prohibitif}

L'obligatif négatif est marqué à l'inaccompli par l'indice de polarité di et sa variante -y. La forme di est le marqueur à toutes les personnes sauf à la première personne où elle est interchangeable avec sa variante $-y$.

\subsubsection{Le mode subordinatif}

\subsubsection{Le temporel négatif}

\begin{tabular}{|l|l|}
\hline \multicolumn{1}{|c|}{ Accompli } & \multicolumn{1}{c|}{ Inaccompli } \\
\hline $\begin{array}{l}\text { bi / ba ma bindul bataaxal } \\
\text { «Quand je n'écris pas de } \\
\text { lettre }\end{array}$ & $\begin{array}{l}\text { bi / ba ma dul bind bataaxal } \\
\text { Quand je n'avais pas l'habitude d'écrire de } \\
\text { lettre » }\end{array}$ \\
\hline
\end{tabular}

$43 \mathrm{Au}$ temporel négatif, la catégorie aspectuelle «inaccompli» est formellement matérialisée par le morphème dul occupant toujours la position antéposée du radical 
verbal, tandis que la catégorie aspectuelle «accomplie» est exempte de toute marque formelle :

\begin{tabular}{|c|c|}
\hline Accompli & Inaccompli \\
\hline bim / bam ...u & bim / bam dul \\
\hline
\end{tabular}

\subsubsection{L'hypothétique négatif}

\begin{tabular}{|c|c|}
\hline Accompli & Inaccompli \\
\hline buñu / suñu bindul bataaxal & buñu / suñu dul bind bataaxal \\
\hline
\end{tabular}

A l'instar du temporel négatif, l'hypothétique négatif est formellement reconnaissable, à l'inaccompli, au morphème dul, antéposé au radical verbal alors qu'à l'accompli, l'hypothétique négatif est morphologiquement non marqué.

\section{Combinaison de l'inaccompli avec d'autres modalités}

Le morphème marqueur de l'aspect inaccompli et sa variante peuvent faire l'objet d'une combinaison avec d'autres éléments grammaticaux tels que la modalité du parfait, les formes temporelles exprimant le passé (indéterminé et habituel) et la marque de négation.

\section{1 Combinaison inaccompli et marque du parfait}

\begin{tabular}{|l|l|l|l|}
\hline $\begin{array}{l}\text { *04-di-na } \\
\text { inacc-pft.3sg }\end{array}$ & $\begin{array}{l}\text { dem } \\
\text { partir }\end{array}$ & $\begin{array}{l}\text { Màkka } \\
\text { Mecque }\end{array}$ & $\begin{array}{l}\text { ren } \\
\text { cette année }\end{array}$ \\
\hline « Il partira pour la Mecque cette année » \\
\hline
\end{tabular}

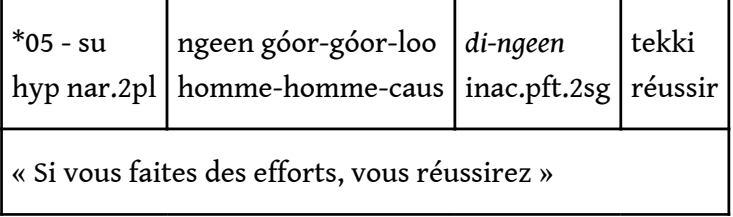

\subsection{Combinaison inaccompli et marque du passé indéterminé (-oon)}

\begin{tabular}{|l|l|l|l|}
\hline $\begin{array}{l}\text { *06- da-ma* } \\
\text { emph.v.1sg }\end{array}$ & $\begin{array}{l}\text { d-oon } \\
\text { inacc-pas ind }\end{array}$ & $\begin{array}{l}\text { dem } \\
\text { partir }\end{array}$ & $\begin{array}{l}\text { tool } \\
\text { champ }\end{array}$ \\
\hline
\end{tabular}




\begin{tabular}{|l|l|l|}
$\begin{array}{l}\text { *07 - tool laa } \\
\text { champ emph.c.1sg }\end{array}$ & $\begin{array}{l}\text { d-oon } \\
\text { inacc-pas ind }\end{array}$ & $\begin{array}{l}\text { dem } \\
\text { partir }\end{array}$ \\
\hline «C'est pour le champ que je partais »
\end{tabular}

\subsection{Combinaison inaccompli et marque du passé habituel (-aan)}

\begin{tabular}{|l|l|l|l|}
\hline $\begin{array}{l}\text { *08 - d-aan } \\
\text { inacc.pas hab }\end{array}$ & $\begin{array}{l}\mathrm{Na} \\
\mathrm{pft} .3 \mathrm{sg}\end{array}$ & $\begin{array}{l}\text { bay } \\
\text { cultiver }\end{array}$ & $\begin{array}{l}\text { dugub } \\
\text { mil }\end{array}$ \\
\hline \multirow{3}{*}{ "Il avait l'habitude de cultiver du mil » }
\end{tabular}

\begin{tabular}{|l|l|l|l|}
\hline $\begin{array}{l}\text { *09- maa } \\
\text { emph.s.1sg }\end{array}$ & $\begin{array}{l}\text { fi d-aan } \\
\text { loc inacc-pas hab }\end{array}$ & $\begin{array}{l}\text { bay } \\
\text { cultiver }\end{array}$ & $\begin{array}{l}\text { ceeb } \\
\text { riz }\end{array}$ \\
\hline "C'est moi qui y cultivais habituellement de l'arachide » \\
\hline
\end{tabular}

\subsection{Combinaison inaccompli marque de négation et marque du passé} indéterminé (-oon)

\begin{tabular}{|l|l|l|l|l|}
\hline $\begin{array}{l}*_{10}-\text { moo } \\
\text { emph.s.3sg }\end{array}$ & $\begin{array}{l}d \text {-oon-ul } \\
\text { inacc-pas ind-neg }\end{array}$ & julli-ji ca & jàkka & $\mathrm{j}-\mathrm{a}$ \\
prier-dir & prep & mosquée def.eloi \\
\hline
\end{tabular}

«C'est lui qui n'avait pas l'habitude d'aller prier à la mosquée »

\begin{tabular}{|c|c|c|}
\hline$*_{11}-d$-oon-uleen & fi & jaar \\
\hline inacc-pas ind-neg.2pl & loc & passer par \\
\hline
\end{tabular}

3.5. Combinaison inaccompli marque de négation et marque du passé habituel (aan)

\begin{tabular}{|l|l|l|}
\hline $\begin{array}{l}*_{12} \text { - mboq laa } \\
\text { maïs emph.s.1sg }\end{array}$ & $\begin{array}{l}\text { d-aan-ul } \\
\text { nacc-pas hab-neg.3sg }\end{array}$ & $\begin{array}{l}\text { bay } \\
\text { i cultiver }\end{array}$ \\
\hline «C'est du maïs que je n'avais pas l'habitude de cultiver » \\
\hline
\end{tabular}

\begin{tabular}{|l|l|l|}
\hline $\begin{array}{l}\text { *13- } \text { - } \text {-aan- } u l \\
\text { inacc-pas hab-neg.3sg }\end{array}$ & $\begin{array}{l}\text { ji } \\
\text { semer }\end{array}$ & $\begin{array}{l}\text { mboq } \\
\text { maïs }\end{array}$ \\
\hline «D'habitude, il ne semait pas du maïs » \\
\hline
\end{tabular}




\section{Valeurs de l'inaccompli}

L'inaccompli correspond à une action, une suite d'actions ou à un état dont l'aboutissement n'est pas atteint au moment de l'énonciation. Compte tenu de son sémantisme qui ne met aucun terme à l'action ou à l'état exprimé, il renferme trois valeurs fondamentales :

\section{- la valeur cursive}

\begin{tabular}{|l|l|l|l|l|}
$\begin{array}{l}* 14-\mathrm{mu} \\
\text { léegi nar.3sg }\end{array}$ & $\begin{array}{l}\text { ngi-y } \\
\text { pres.inacc pleurer }\end{array}$ & $\begin{array}{l}\text { jooy } \\
\text { prep }\end{array}$ & $\begin{array}{l}\text { ca } \\
\text { matin }\end{array}$ & $\begin{array}{l}\text { suba ba } \\
\text { jusqu'à maintenant }\end{array}$ \\
\hline
\end{tabular}

" Il pleure depuis ce matin »

\begin{tabular}{|l|l|l|}
\hline $\begin{array}{l}* 15-\text { dafay } \\
\text { emph.v.3sg-inacc }\end{array}$ & $\begin{array}{l}\text { dem } \\
\text { aller }\end{array}$ & $\begin{array}{l}\text { tefes } \\
\text { plage }\end{array}$ \\
\hline
\end{tabular}

\section{- la valeur de l'habitude}

\begin{tabular}{|l|l|}
\hline $\begin{array}{l}* 16-\text { damay } \\
\text { emph.v.1sg inacc }\end{array}$ & $\begin{array}{l}\text { tux } \\
\text { fumer }\end{array}$ \\
\hline «Je fume habituellement
\end{tabular}

\begin{tabular}{|l|l|l|}
\hline $\begin{array}{l}* 17-\text { bëccëg } \\
\text { journée }\end{array}$ & $\begin{array}{l}\text { lañuy } \\
\text { emph.c.1pl .inacc }\end{array}$ & $\begin{array}{l}\text { añ } \\
\text { déjeuner }\end{array}$ \\
\hline «C'est dans la journée qu'on déjeune d'habitude » \\
\hline
\end{tabular}

\begin{tabular}{|l|l|l|}
\hline $\begin{array}{l}\text { *18 - dafay } \\
\text { emph.v.3sg-inacc }\end{array}$ & $\begin{array}{l}\text { daanu } \\
\text { tomber }\end{array}$ & $\begin{array}{l}\text { esprit } \\
\text { esprit }\end{array}$ \\
\hline
\end{tabular}

\begin{tabular}{|l|l|l|}
\hline $\begin{array}{l}* 19-\text { damay } \\
\text { emph.v.1sg inacc }\end{array}$ & $\begin{array}{l}\text { xiif } \\
\text { avoir.fain }\end{array}$ & $\begin{array}{l}\text { guddi } \\
\text { nuit }\end{array}$ \\
\hline «J'ai l'habitude d'avoir faim la nuit » \\
\hline
\end{tabular}

\begin{tabular}{|l|l|l|l|l|l|}
\hline $\begin{array}{l}*_{20}-\text { dina-ñu } \\
\text { fut.1pl }\end{array}$ & $\begin{array}{l}\text { di } \\
\text { inacc }\end{array}$ & $\begin{array}{l}\text { bind-ante } \\
\text { écrire-rec }\end{array}$ & $\begin{array}{l}\text { altine } \\
\text { lundi }\end{array}$ & $\begin{array}{l}\text { ju } \\
\text { rel }\end{array}$ & $\begin{array}{l}\text { ne } \\
\text { être }\end{array}$ \\
\hline
\end{tabular}


« Nous nous écrivons chaque lundi »

49

\section{- la valeur projective}

\begin{tabular}{|c|c|c|}
\hline $\begin{array}{l}* 21 \text { - dinaa } \\
\text { fut.1sg }\end{array}$ & $\begin{array}{l}\text { jàng } \\
\text { apprendre }\end{array}$ & $\begin{array}{l}\text { wolof } \\
\text { wolof }\end{array}$ \\
\hline $\mathrm{J}$ & le wolof & \\
\hline
\end{tabular}

\begin{tabular}{|l|l|l|}
\hline $\begin{array}{l}*_{22}-\text { suba } \\
\text { demain }\end{array}$ & $\begin{array}{l}\text { la-ay } \\
\text { emph.c.3sg-inacc }\end{array}$ & $\begin{array}{l}\text { liggéey } \\
\text { travailler }\end{array}$ \\
\hline \multicolumn{2}{|l|}{} \\
«C'est demain que je travaillerai »
\end{tabular}

Au terme de cette analyse de l'opposition aspectuelle accompli / inaccompli en wolof contemporain, nous avons relevé que l'expression formelle de l'aspect s'oppose à une marque zéro et produit un système binaire avec un pôle accompli non marqué ou constituant un amalgame de catégories sémantiques difficilement analysable synchroniquement, et un inaccompli formellement identifiable par le morphème di et ses différentes variantes, $d$ - et $-y$.

51 En effet, le marqueur di apparaît postposé aux marques de personne et antéposé au radical verbal, en règle générale. Cet ordre syntaxique est strict. Le morphème $d i$ exprime la polarité « inaccompli » à :

- toutes les personnes du narratif affirmatif,

- toutes les personnes du parfait affirmatif,

- toutes les personnes de l'emphatique du sujet affirmatif,

- la 2pl de l'emphatique du verbe affirmatif,

- la 2pl de l'emphatique du complément affirmatif,

- toutes les personnes de l'impératif affirmatif,

- la 2pl du temporel affirmatif,

- la 2sg, la 3sg et à la 2pl de l'hypothétique affirmatif,

- toutes les personnes du parfait négatif avec sa variante $d$-,

- toutes les personnes de l'impératif négatif,

- toutes les personnes de l'obligatif négatif.

La variété $-y$ toujours suffixée à la marque de personne constitue le marqueur inaccompli de :

- la 2sg, la 3sg et la 3pl de l'emphatique du verbe affirmatif,

- la 2sg, la 3sg et la 3pl de l'emphatique du complément affirmatif,

- toutes les personnes de l'obligatif affirmatif où il est interchangeable avec di sauf à la $2 \mathrm{pl}$ où ce dernier apparait seul,

- toutes les personnes du temporel affirmatif sauf la 2pl marquée par le morphème di,

- la 1sg et les 1 pl et 3pl de l'hypothétique affirmatif,

- la 1sg interchangeable avec di au parfait négatif,

- la 1sg de l'obligatif négatif interchangeable avec di. 
La forme segmentale $-y$ est le marqueur inaccompli de toutes les personnes au présentatif affirmatif.

La forme segmentale $d$ - est le traducteur inaccompli de :

- la 1 sg du parfait négatif où elle peut être combinée avec -y ou di et de toutes les autres personnes de ce paradigme où elle est combinée avec di,

- de toutes les personnes de l'emphatique du sujet négatif,

- de toutes les personnes de l'emphatique du verbe négatif,

- de toutes les personnes de l'emphatique du complément négatif,

- de toutes les personnes du temporel négatif,

- de toutes les personnes de l'hypothétique négatif.

La voyelle finale $-u$ de le troisième personne du singulier du temporel, des première et troisième personnes du pluriel du parfait, de l'obligatif, du temporel et de l'hypothétique tombent régulièrement (dialectalement : saloum).

\section{BIBLIOGRAPHIE}

CISSE M., Éléments systématiques du wolof contemporain, Dakar, CLAD, 1983, 85p.

CREISSELS D., Éléments de syntaxe générale, Paris, PUF, 1995, 332p.

CISSE M., Unités et catégories grammaticales du wolof, Paris, NE, 1998, 324p.

CISSE M., Dictionnaire Français-Wolof, Paris, Langues \& Mondes, L’Asiathèque, 2007, p. 389.

CHURCH E., Le système verbal du wolof, Département de Linguistique Générale et de Langues Négro-Africaines de la Faculté des Lettres et des Sciences Humaines, Université Cheikh Anta Diop de Dakar, 1981, 365p.

DIALO A., Système verbal et conjugaison du wolof? , Université Cheikh Anta Diop de Dakar, Faculté des Lettres et Sciences Humaines, Département de Linguistique et Sciences du Langage, TBR $n^{\circ} \mathrm{V}$, 2012.

FEUILLET J., Introduction à la typologie linguistique, Paris, Honoré Champion, 2006, 720p.

ROBERT S., Système verbal et énonciation en wolof, Thèse de doctorat de $3^{\text {ème }}$ cycle, Université de Paris 7, 1989, 397p.

SAUVAGEOT S., Description synchronique d'un dialecte wolof : le parler du dyolof, Dakar, IFAN, 1965, 274p.

VOISIN-NOUGUIER S., Relations entre fonctions syntaxiques et fonctions sémantiques en wolof, Thèse de doctorat, Université Lumière Lyon 2, Faculté des Lettres, Sciences du Langage et Arts,

Département Sciences du Langage, 2002, 371p.

\section{ANNEXES}




\section{ABREVIATIONS utilisées dans l'analyse des exemples:}

caus : causatif.

def.proc : défini proche.

def.eloi : défini éloigné.

dir : directionnel.

Emph.c : emphatique du complément.

emph.s : emphatique du sujet.

emph.v : emphatique du verbe.

fut : futur.

hyp : hypothétique.

inacc : inaccompli.

loc : locatif.

nar : narratif.

neg : négatif.

$o$ : indice objet.

part : partitif.

pas hab : passé habituel.

pas ind : passé indéterminé.

pft : parfait.

pos : possessif.

prep : préposition.

pres : présentatif.

rec : réciproque

rel : relatif

$1 \mathrm{sg}: 1^{\text {ère }}$ personne du singulier

$3 \mathrm{sg}: 3^{\text {ème }}$ personne du singulier

$2 \mathrm{pl}: 2^{\text {ème }}$ personne pluriel

\section{NOTES}

1. Le terme complément renvoie, dans l'énoncé, à tout constituant qui ne joue pas le rôle de sujet ou de verbe en relation prédicative.

\section{RÉSUMÉS}

Comment est cryptée la polarité aspectuelle en wolof contemporain? Poser de la sorte la problématique de l'aspect dans cette langue et tenter d'y apporter un éclairage méthodique c'est aussi contribuer à élucider certaines zones d'ombre encore persistantes dans l'approche du système verbal de celle-ci. Comment, dans une langue aussi agglutinative comme le wolof, identifier et isoler des morphèmes sans recourir à un examen diachronique parsemé d'incohérence ? Nous tentons de présenter la manifestation de l'opposition accompli/inaccompli à travers les différents paradigmes de conjugaison; car pour comprendre le système verbal du 
wolof, il est essentiel de se faire une idée claire du rôle de l'aspect. Cette analyse s'intègre dans la dialectologie du wolof et se veut aussi un apport aux études typologiques.

How the aspect polarity is encoded in contemporary Wolof? Tackling such an issue and trying to bring a methodological clarification on it, implies a contribution to a better understanding of Wolof verbal system. How to identify and isolate morphemes in an agglutinative language like Wolof, without resorting to a diachronic analysis, somewhat full of incoherence? In this article, we will introduce the dichotomy achieved/unachieved throughout the different Wolof conjugation paradigms in order to shed light on some blurred aspects of the topic. For a better understanding of Wolof verbal system, it is compulsory to have a clear idea of the role played by aspect. Such an approach is also a contribution to typological as well as dialectological studies.

\section{INDEX}

Mots-clés : wolof, polarité aspectuelle, accompli/inaccompli, système verbal

Keywords : wolof, aspectual polarity, achieved/unachieved, verbal system

\section{AUTEUR}

\section{MAMADOU CISSÉ}

Faculté des Lettres et Sciences Humaines Université Cheikh Anta Diop - Dakar - Sénégal 